\title{
An Expedited WiFi Connection Establishment Scheme for Railroad Wireless Communications
}

\author{
Kwang Kiun Yoo and Ronny Yongho Kim* \\ Department of Railroad Electrical and Electronics Engineering, \\ Korea National University of Transportation, Uiwang, Gyeonggi, 437-763 Korea
}

ronnykim@ut.ac.kr

\section{Abstract}

Always on Internet service has become norm and people are abtêto-njoy anytime, anywhere broadband wireless communication services. With the emergende of smart phone and tablet, wireless network traffic has increased exponentially, Wiveless network traffic is expected to continuously increase dramatically as move and more communication capable multimedia devices are expected to be connected to wireless network. In order to offload heavy traffic of cellular network, WiFi has chosen since WiFi normally operates in license exempt bands. 3rd Generation Partnership Program (3GPP) has started study item on 3GPPWiFi interworking in order to alleviate heayy traffic problem. Since WiFi is independent and different wireless technology, 3GPP nêeds to be specially designed to provide seamless and high quality interworking with WiFt. Long Term Eyolution (LTE)-WiFi interworking has a technical challenge of WiFi network detection. A good solution should provide not only fast WiFi network detection but also battery power saving of mobile phones. In this paper, we propose LTE and WiFi interworking scheme for railroad wireless communication. Our solution is able to provide both expedited WiFi connection establishment and battery power saving by exploiting cuntque characteristics of railroad communication.

Keywords: Railroad Wiceless Communication, WiFi, IEEE 802.11, 3GPP LTE, IT Convergence

\section{Introduction}

As Information and Communication Technology (ICT) evolves, always on Internet service has become possible and people are able to enjoy anytime, anywhere broadband wireless communication services. In order to provide broadband services International Telecommunication Union (ITU) standardized 4G wireless standard called IMT-Advanced [1]. HEEE 802.16 working group and 3rd Generation Partnership Program (3GPP) developed the standards [2][3] in order to meet or even exceed the IMT-Advanced requirements.

WiFi, which employs IEEE 802.11 air interface standard [4] is a wireless access service widely used nowadays. Since WiFi typically utilizes license exempt band such as $2.4 \mathrm{GHz}$ Industrial Scientific and Medical (ISM) band, WiFi can be deployed freely by users. Such simple deployment makes WiFi popular and easy WiFi access possible. As multimedia services have become popular Internet services, Internet packets through wireless networks get larger. Therefore, cellular network such as 3GPP LTE/LTE-A is seeking a solution to offload its traffic to WiFi using license exempt band. There are several challenges in WiFi data offloading. One of major challenges of 3GPP-WiFi interworking is WiFi network

${ }^{*}$ Ronny Yongho Kim is the corresponding author. 
detection. Since WiFi is a different radio access technology (RAT) from 3GPP, WiFi operation is independent from 3GPP network operation. While WiFi/Cellular (LTE/LTE-A) dual mode mobile terminals are working in 3GPP mode, it is not easy to discover WiFi network because WiFi detection is done with blind search. Such blind WiFi search consumes substantial amount of battery power. In order to alleviate WiFi detection problem in a standardized way, 3GPP started a study item to provide efficient 3GPP-WiFi interworking mechanisms.

Variety of wireless technologies, e.g., Terrestrial Trunked Radio (TETRA) [5], GSM-R [6], IEEE 802.11 [4], etc., are used for railroad communications. Railroad wireless communication has its unique characteristics since train trajectory and time are predetermined. People can enjoy broadband Internet service while traveling by train due to broadband wireless access technologies like WiFi and LTE/LTE-A. If unique characteristics of railroad wireless communication can be properly considered, a very eff cient 3GPP-WiFi interworking scheme can be developed for railroad wireless dommunieations. In this paper, we propose an efficient railroad wireless communication scheme using 3GPP-WiFi interworking. With the proposed scheme, better quality wireless services can be provided to train passengers. A preliminary result of this paper was presented in [7].

This paper is organized as follows. In Section 2, railroad wireless communication and current 3GPP-WiFi interworking are reviewed. In Section 3, a novel expedited WiFi connection establishement scheme using $3 \mathrm{GPP} W i F i$ interworking is proposed and in depth discussion on the proposed scheme is proyided. A norel protocol architecture to support the proposed scheme is also proposed in Section 3. InSection 4, finally, we conclude the paper.

\section{Railroad Wireless Communication}

\subsection{Railroad Wireless Network Confíguration}

In typical railroad wreless communication network configuration, cellular (LTE/LTE-A) terminals and WiFi terminals of train passengers (users) are located within the train and they communicate either with LTE celluarbase station or with WiFi Access Point (AP). On board terminal is able to provide a tethering service using 3GPP LTE and WiFi to provide passengers with WiFi broadband Internet access. Since Voice over LTE (VoLTE) [8] can be provided, all railroad wreless communication features and services can be provided using only LTE.

Railroad communication network has its unique network configuration since train moves along fixed railroad. A train which consists of multiple train cars moves along a train track. Since railroad communication network is a linear network and train's movement is prescheduled, communication of train passengers or crews can be easily managed.

\subsection{LTE and WiFi Interworking}

WiFi is a very popular wireless access scheme nowadays, since WiFi typically utilizes license exempt band making easy access possible. WiFi is chosen by $3 \mathrm{GPP}$ to offload heavy traffic of cellular system like 3GPP LTE. One of major challenges of 3GPP-WiFi interworking is WiFi network detection. Since WiFi is a different RAT from 3GPP, WiFi operation is independent from 3GPP network operation. While WiFi/Cellular (LTE/LTE-A) mobile terminals are working in 3GPP mode, it is not easy to discover WiFi network because WiFi detection is done with blind search. Such blind WiFi search consumes substantial amount of battery power. In order to alleviate $\mathrm{WiFi}$ detection problem in a standardized way, $3 \mathrm{GPP}$ started a study to provide efficient $3 \mathrm{GPP}-\mathrm{WiFi}$ interworking mechanisms. At the time of writing this paper, 3GPP Radio Access Network (RAN) 2 working group has three 
solutions [9]: 1. Periodic 3GPP system information broadcast with WiFi network discovery information, 2. On demand delivery of 3GPP control message WiFi network discovery information, 3. Measurement based WiFi data offloading.

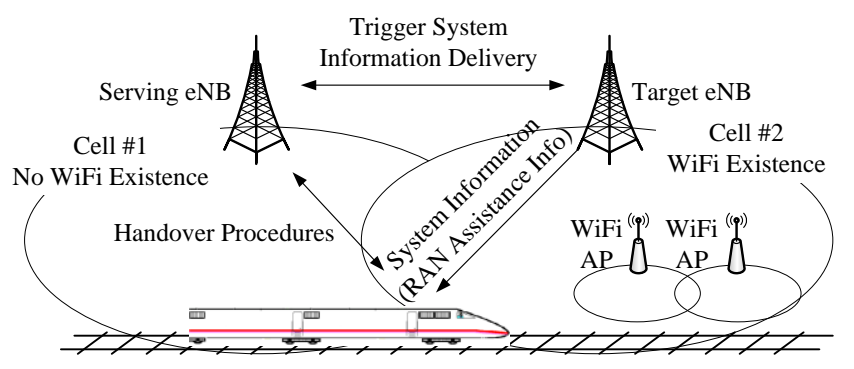

Figure 1. RF-CBTC Network Configuration

In railroad wireless communication environment, LTE-WiFi overlay network can be configured. While a train is moving, LTE commun ication link is used since LTE provides a wide coverage. However, while a train is staying at train stations for passenger boarding and deboarding, it is beneficial for an on board ralroad communication unit to utilize WiFi communication link by accessing APs installed at the train stations. Also substantial number of deboarding passengers will try to connect to WiFi APs at the deboarding stations. Since the WiFi stations (STAs) of deboarding passengers do not have WiFi APs' access information, it will take substantial amount of time for WiFi STAs to discover WiFi APs and establish WiFi links. The other technical challenges of 3GPP-WiFi interworking are seamless interworking and fast handover and transition from 3GPP network to WiFi network.

\section{Expedited WiFi Connection Establishment Scheme for Railroad Wireless Communication}

\subsection{Railroad Wireless Communication using LTE and WiFi Interworking}

Figure 1 shows an exemplaty scenario to explain our proposed scheme. When the train performs handover to the cell where a train station is located, the current serving base station (eNB) notifies the targeteNB of a train's handover. After receiving the notification from the serving eNB, the target eNB is able to include RAN assistance information, which can be used for WiFil detection, in the system information. Upon reception of such system information including WiFi detection information, the on board terminal is able to detect WiFi network very easily within short period of time using the received WiFi detection information. Since the WiFi module of the on board terminal is triggered to search WiFi upon receiving the RAN assistance information, battery power can be saved substantially. When the train performs handover to the other cell, the eNB is able to exclude RAN assistance information from the system information. In this way, the eNB can include RAN assistance information in the system information only when it is required, i.e., only when there exist mobile terminals to receive the information.

3GPP network should be aware of mobile's WiFi connection profile including WiFi networks to which mobile wishes to connect. Since railroad wireless communication network is specially designed for railroad, it is not a difficult problem for the wireless network to manage railroad wireless terminals' WiFi connection profile. Since system information broadcast requires much more radio resources than unicast control message delivery, RAN assistance information delivery using system information is very costly. However, our 
proposed scheme provide efficient way to use radio resources by including RAN assistance information in system information whenever it is only necessary, i.e., entrance of train into the cell.

\subsection{Proposed Protocol Architecture for Railroad Wireless Communication}

In order to provide a $3 \mathrm{GPP}-\mathrm{WiFi}$ interworking function, new protocol architecture needs to be designed. We employ a similar protocol architecture to the one proposed in IEEE 802.21 standard [10]. A virtualization layer is placed above WiFi's and LTE's MAC layer in order to provide communication between different communication modules. LTE Radio Resource Control (RRC), Radio Link Control (RLC) and Medium Access Control (MAC) are represented with LTE MAC for simplicity. Inter-protocol communication can be realized with virtual layer (VL) service access point (SAP). Primitives are delivered to the $\mathrm{VL}$ through SAPs. The VL translates primitives of one communication module into primitives of another communication module.

After handover, LTE protocol module receives system information including RAN assistance information, i.e., WiFi detection information. LTE MAC, actually LTE RRC without simplification, extracts WiFi detection information and transmits it using primitives to virtualization layer. Then, the virtualization ayyer translates LTE's primitive into WiFi's primitive and delivers the translated primitive to the WiFPMAC. WiFi MAC is able to commence $\mathrm{WiFi}$ detection procedure using the information received from the LTE communication module. In order to provide seamless and fast interworking, WiFi connection status is transmitted to the LTE communication module by using the same mechanism through the virtualization layer.

\subsection{Proposed Protocol Procedure for Railroad Wireless Communication}

A detailed proposed proeedure for ralload wireless communication using LTE and WiFi interworking is shown in Figure 2 m order to minimize the impact on 3GPP standard, most of the existing procedure is reused. Typically handover is triggered by checking the measurement report from, the $\mathscr{E}$. When the measured signal qualities of the source and target eNB meet certain criteria, handover decision is made and handover procedure is triggered by the source eNB. Upon(reception of handover request, the target eNB can be notified that a train will be handed oyer to its cell by checking the UE's identification (ID). After the completion of handover procedure by receiving RRC Connection Configuration Complete control message, the target eNB which becomes the new source eNB, transmits system information including RAN assistance information. Additionally, the target eNB can delay the delivery of the system information until the train comes to the train station which is within the arget eNB's cell coverage. Delivery time can be easily calculated since the train's speed and trajectory is predetermined. Upon receiving system information, RAN assistance information (WiFi detection information) is delivered to the WiFi communication module using the scheme described in the previous sub-section. WiFi detection information delivery to the WiFi communication module triggers WiFi detection procedure. Since the WiFi communication module already received WiFi detection information, WiFi scanning procedure (passive scanning or active scanning) can be omitted and commence WiFi network connection procedure immediately which saves substantial amount of time. 


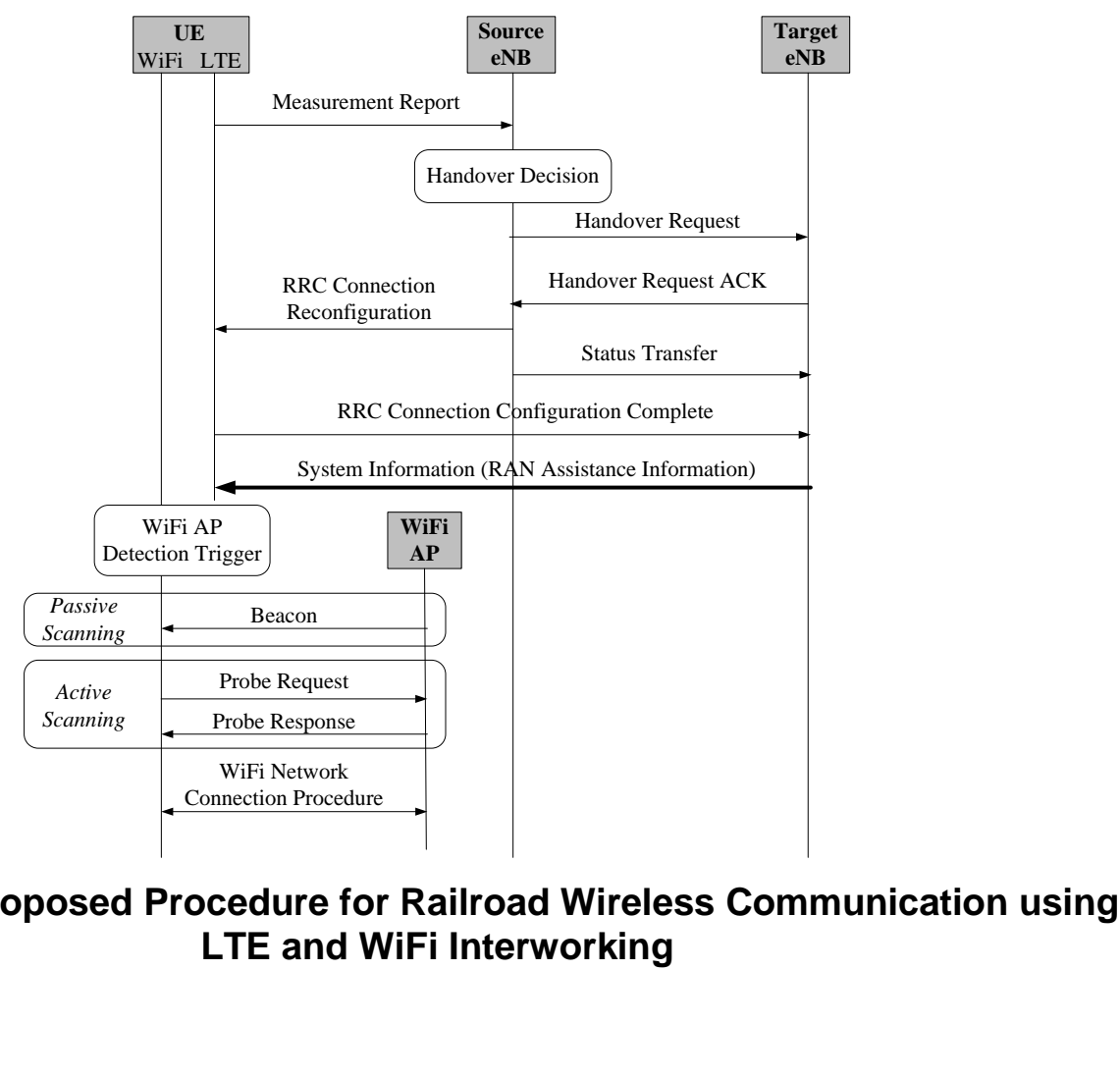

Figure 2. A Proposed Procedure for Railroad Wireless Communication using LTE and WiFi Interworking

\section{Conclusion}

In this paper, an expedited WiFiconnection establishment scheme using LTE and WiFi interworking is proposed. Sinceuheavy wireless network traffic is very serious problem, 3GPP has been seeking for a solution to traffic offloading. Very promising solution is 3GPP-WiFi interworking. Railroad wireless communication is expected to use LTE technology together with WiFi and has very unique railroad wireless network configuration. By taking the advantage of unique railroad wireless network configuration, a very eificient LTE-WiFi interworking scheme for railroad wireless communication can be designed. Since our proposed scheme not only provides expedited WiFi comnection establishment but also battery power efficient WiFi operation, railroad wireless users' experience is expected to be enhanced substantially.

\section{Acknowledgements}

This Tesearch was supported by Basic Science Research Program through the National Researeh Foundation of Korea (NRF) funded by the Ministry of Education, Science and Technology(NRF-2012R1A1A1014610).

\section{References}

[1] ITU-R M.2134, "Requirements Related to Technical System Performance for IMT-Advanced Radio Interface(s) [IMT.TECH]", draft new report, (2008) November.

[2] "IEEE Standard for Local and metropolitan area networks Part 16: Air Interface for Broadband Wireless Access Systems Amendment 3: Advanced Air Interface”, IEEE Std 802.16m-2011, (2011) May 12.

[3] 3GPP Technical Specification 36.300, "E-UTRA and E-UTRAN Overall Description Stage 2 (Release 10)", www.3gpp.org. 
[4] "IEEE Standard for Information Technology-Telecommunications and information exchange between systems Local and metropolitan area networks--Specific requirements Part 11: Wireless LAN Medium Access Control (MAC) and Physical Layer (PHY) Specifications”, IEEE Std 802.11-2012, (2012).

[5] “Terrestrial Trunked Radio (TETRA). Voice plus Data (V+D), Part 2: Air Interface (AI)," ETSI EN 300 39202, v3.2.1 (2007).

[6] UIC, "GSM-R Procurement Guide," (2007) February.

[7] K. K. Yoo and R. Y. Kim, "Efficient railroad wireless communication scheme using 3GPP LTE and WiFi interworking," Proceedings of the Workshop on Mobile and Wireless, (2013) December 11-13, Jeju, Korea.

[8] 3GPP Technical Specification 22.468: "Group Communication System Enablers for LTE (GCSE_LTE) (Release 12)", (2013) June.

[9] 3GPP Technical Report 37.834, "Technical Specification Group Radio Access Network; Study on WLAN/3GPP Radio Interworking (Release 12), (2013) May.

[10] "IEEE Standard for Local and metropolitan area networks- Part 21: Media Independent Handover Seryices," IEEE Std 802.21-2008, (2009).

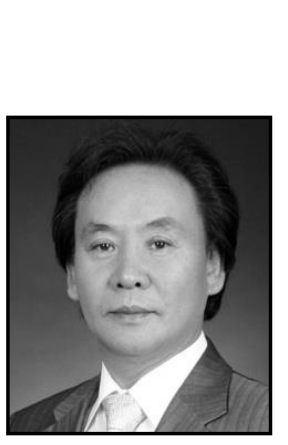

Authors

Kwang Kiun Yoo, he received his Ph.D. degred from Kwangwoon University, Seoul, Korea, in 1997. He became a Fellow Member of Institute of Railway Signal Engineers in 2007. Since 1992, he has been with the Department of Railrøad Electrical and Electronics Engineering, Korea National Univensity of Transportation (KNUT), Gyeonggi, Korea, ythere he is a Professor. Since 2012, he has been a dean of college of rallroad sciences in KNUT. His research interests are railroad sighal engineering, communication based train control and railroad engineering

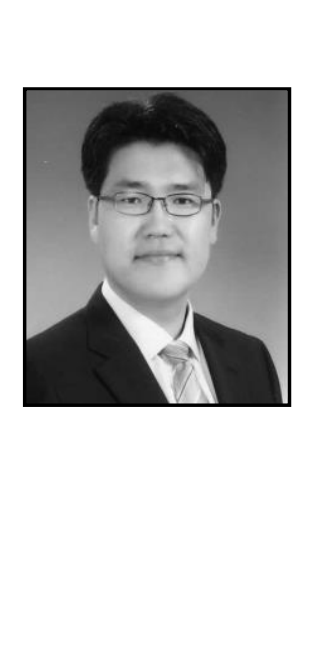

Ronny Yongho Kim, he received his Ph.D. degree from Yonsei Uniyersity, Sebul, Korea, 2010. He became an IEEE Senior Member in 2010. From 1998 to 2010, he was with LG Electronics. Since 2012 he has been with the Department of Railroad Electrical and Electronies Engineering, Korea National University of Transportation (KNUT), Gyeonggi, Korea, where he is an Assistant Propessor. His current research interests are railroad communications, wireless networks, IT convergence and machineto-machine communications. 